\title{
Effects of Task Performance Symmetry on Human Collective Decision-Making ${ }^{\dagger}$
}

\author{
Malte Lau Petersen * and Lars Bach \\ Interacting Minds Center, University of Aarhus, 8000 Aarhus C, Denmark; lars4bach@gmail.com \\ * Correspondence: maltelau@protonmail.com \\ + Presented at Symmetry 2017-The First International Conference on Symmetry, Barcelona, Spain, \\ 16-18 October 2017.
}

Published: 12 January 2018

In human joint decision-making, a prevalent question is whether the two persons could have solved the tasks equally well on their own, or possibly better as individual task-decision-makers? When allowed to communicate and hence combine perceived sensory information among individuals in a group, previous studies have indicated that symmetry in task performance between subjects promotes a synergetic performance effect. In other words, two heads are better than one when there is equality or symmetry in perceptual sensitivity and task performance. However, when task performance is asymmetrical between individuals, the collective performance is actually worse than the better performing individual. Similar findings exist with language symmetry: contextspecific alignment can lead to collective benefit but, e.g., indiscriminate repetitions of the interaction partner's words can worsen the collective performance. This study deals with the comparison of results from different sensory modalities and hence addresses whether or not this effect seems domain specific or presents a robust general pattern across sensory modi. Two analyses (Frequentist and Bayesian) are employed since previous studies utilize the frequentist approach and hence allow us to make a comparison. The Bayesian analysis, however, allows us to explicitly specify all assumptions, carry the uncertainty all the way through the model, and directly compare the predictive power of the proposed effects.

Conflicts of Interest: The authors declare no conflict of interest.

(C) 2018 by the authors. Licensee MDPI, Basel, Switzerland. This article is an open access article distributed under the terms and conditions of the Creative Commons Attribution (CC BY) license (http://creativecommons.org/licenses/by/4.0/). 